\title{
Wogonin Attenuates Ovalbumin Antigen-Induced Neutrophilic Airway Inflammation by Inhibiting Th17 Differentiation
}

\author{
Rie Takagi, ${ }^{1}$ Masaaki Kawano, ${ }^{1}$ Kazuyuki Nakagome, ${ }^{2,3}$ Kumiko Hashimoto, ${ }^{1}$ \\ Takehiro Higashi, ${ }^{1}$ Katsuya Ohbuchi, ${ }^{4}$ Atsushi Kaneko, ${ }^{4}$ and Sho Matsushita ${ }^{1,3}$ \\ ${ }^{1}$ Department of Allergy and Immunology, Faculty of Medicine, Saitama Medical University, Saitama 350-0495, Japan \\ ${ }^{2}$ Department of Respiratory Medicine, Saitama Medical University, Saitama 350-0495, Japan \\ ${ }^{3}$ Allergy Center, Saitama Medical University, Saitama 350-0495, Japan \\ ${ }^{4}$ Tsumura Research Laboratories, Tsumura \& Co., Ibaraki 300-1192, Japan
}

Correspondence should be addressed to Atsushi Kaneko; kaneko_atsushi@mail.tsumura.co.jp

Received 20 March 2014; Accepted 9 May 2014; Published 28 May 2014

Academic Editor: B. L. Slomiany

Copyright (C) 2014 Rie Takagi et al. This is an open access article distributed under the Creative Commons Attribution License, which permits unrestricted use, distribution, and reproduction in any medium, provided the original work is properly cited.

\begin{abstract}
Allergic airway inflammation is generally considered to be a Th2-type immune response. Recent studies, however, have demonstrated that Thl7-type immune responses also play important roles in this process, particularly in the pathogenesis of neutrophilic airway inflammation, a hallmark of severe asthma. We scrutinized several Kampo extracts that reportedly exhibit antiinflammatory activity by using in vitro differentiation system of human and mouse naïve $\mathrm{T}$ cells. We found that hange-shashin-to (HST) and oren-gedoku-to (OGT) possess inhibitory activity for Th17 responses in vitro. Indeed, wogonin and berberine, major components common to HST and OGT, exhibit Th17-inhibitory activities in both murine and human systems in vitro. We therefore evaluated whether wogonin suppresses OVA-induced neutrophilic airway inflammation in OVA TCR-transgenic DO11.10 mice. Consequently, oral administration of wogonin significantly improved OVA-induced neutrophilic airway inflammation. Wogonin suppressed the differentiation of naïve T cells to Th17 cells, while showing no effects on activated Th17 cells.
\end{abstract}

\section{Introduction}

Different classes of specific immune responses are driven by the biased development of effector $\mathrm{CD} 4^{+} \mathrm{T}$-cell subsets, that is, T helper 1 (Th1), Th2, and Th17 cells, that activate different components of cellular and humoral immunity. Thcell differentiation is critical for achieving proper immune responses, and imbalances in either the function or activity of these cell types are responsible for many immune diseases, including autoimmunity, cancer, and allergies [1-3]. T-cell receptor stimulation and costimulation allow naïve $\mathrm{T}$ cells to develop into protective effector cells, normally accompanied by the high-level expression of selective sets of cytokines. The balance between these cytokines and the resulting class of immune responses strongly depends on the conditions under which the DCs are primed for the expression of T-cellpolarizing molecules [4].

We recently found that such activities can be scrutinized by assessing mixed lymphocyte reactions (MLR) [5], cAMP
[6], and the differential expressions of Notch ligand isoforms [7]. Of note, MLR meets the needs for such primary screening processes because (i) naive $\mathrm{CD} 4 \mathrm{~T}$ cells are the major population that reacts against allogeneic antigen presenting cells, indicating that the cytokines detected in the cell-culture supernatant fluids of MLR are of newly differentiated CD4T cells; (ii) it is mediated by the physiological MHC-peptideTCR interactions; (iii) the clonal frequency of alloreactive naive CD4T cells is markedly higher than that of foreign antigen-specific naïve CD4T cells; and (iv) alloreactive Th1, Th2, and Th17 cells are induced without the use of externally added cytokines, allowing us to target all the Th subsets in a single cell-culture well. Using these systems, we revealed that the administration of a D1-like-R antagonist inhibits Th17 differentiation, thereby improving clinical phenotypes, including experimental autoimmune encephalomyelitis (EAE), type 1 diabetes, glomerulonephritis, rheumatoid arthritis [8], and neutrophilic airway inflammation [9], in mouse models. 
In order to identify novel compounds that have inhibitory effects on Th17 generation, we focused on a traditional Japanese herbal medicine, Kampo, which is well known to exert various immunomodulatory effects [10-12]. In this report, we screened several Kampo extracts known to possess anti-inflammatory activities and identified two Kampo extracts with Th17-inhibitory activity. One is oren-gedoku-to (OGT), which is ethically prescribed to treat inflammatory diseases, including dermatitis, gastric ulcers, gastritis, and related diseases. The other is hange-shashin-to (HST), which is effective in treating disorders of the digestive mucosa, such as gastritis, diarrhea, and stomatitis. OGT and HST have been studied in several experimental models of gastritis and colitis [13-16]; however, their mechanisms have yet to be determined. Both OGT and HST contain common components originating from two kinds of medicinal herbs, Scutellaria root and Coptis rhizome. A representative flavonoid, wogonin, is one of the primary components of Scutellaria root, and berberine is an isoquinoline alkaloid that is rich in Coptis rhizome. The aim of this study is to identify the active components contained in OGT and HST and to evaluate their immunomodulatory features, particularly with respect to Tcell lineage differentiation.

\section{Materials and Methods}

2.1. Test Samples. Kampo medicines are aqueous extracts of a mixture of natural crude drugs. Eight kinds of Kampo extracts, HST, OGT, HJG (hachimi-jio-gan), SST (shosaiko-to), HET (hochu-ekki-to), JTT (juzen-taiho-to), DKT (dai-kenchu-to), and SRT (sai-rei-to) were obtained from Tsumura and Co. (Tokyo, Japan) in the form of dried powdered extract. Berberine and wogonin were purchased from Wako Pure Chemical Industries, Ltd. (Osaka, Japan). Alternatively, wogonin was extracted from Scutellaria root at Tsumura and Co. at a purity of more than $99.5 \%$ for the in vivo studies.

The Kampo extracts were suspended in dimethyl sulfoxide (DMSO) at $10 \mathrm{mg} / \mathrm{mL}$ and passed through a $0.22 \mu \mathrm{m}$ membrane following gentle centrifugation. The supernatant fluids were added to the cultures at a final concentration of $10 \mu \mathrm{g} / \mathrm{mL}$. The wogonin and berberine were dissolved in DMSO and added to the cultures at the described concentrations.

2.2. Mice. SJL and BALB/c mice were obtained from Charles River Japan, Inc. (Kanagawa, Japan). OVA TCR-transgenic DO11.10 mice were obtained from the Jackson Laboratory (Bar Harbor, ME). All animal experiments were approved by and performed in compliance with the Institutional Animal Care and Use Committee Guidelines.

2.3. Preparation of Human Mo-DCs and T Lymphocytes. Human Mo-DCs and CD45RA ${ }^{+}$naïve $\mathrm{CD}^{+}{ }^{+} \mathrm{T}$ cells $(>99 \%$ purity) were prepared as previously described [9]. Briefly, human Mo-DCs were prepared from human peripheral blood specimens (PBMCs) using positive selection with CD14 MicroBeads (Miltenyi Biotec). The Mo-DCs were then cultured in the presence of GM-CSF $(50 \mathrm{ng} / \mathrm{mL})$ and IL$4(50 \mathrm{ng} / \mathrm{mL})$ for five days. Human $\mathrm{CD}_{45 \mathrm{RA}^{+}}$naïve $\mathrm{CD} 4^{+}$ $\mathrm{T}$ cells were prepared via negative selection using a naïve $\mathrm{CD} 4^{+} \mathrm{T}$-cell isolation kit II (Miltenyi Biotec, Auburn, U.S.A.). The study using PBMCs obtained from healthy volunteers was approved by the Saitama Medical University Ethics Committee.

2.4. Two-Way MLR Assay. For the mouse two-way MLR, $3 \times$ $10^{6}$ spleen cells obtained from SJL/J mice $\left(\mathrm{H}-2^{\mathrm{s}}\right)$ and $3 \times$ $10^{6}$ spleen cells obtained from $\mathrm{BALB} / \mathrm{c}$ mice $\left(\mathrm{H}-2^{\mathrm{d}}\right)$ were mixed and then incubated with Kampo extracts $(10 \mu \mathrm{g} / \mathrm{mL})$, wogoinin $(0.1,0.3$, and $1 \mu \mathrm{M})$, or berberine $(0.1,0.3$, and $1 \mu \mathrm{M})$ in $2 \mathrm{~mL}$ of DMEM medium containing $10 \%$ FCS in a flat-bottomed 12-well plate for seven days. The culture supernatants were assayed for IFN- $\gamma$, IL-4, IL-5, IL-6, and IL17 using ELISA kits (R\&D systems, U.S.A.). For the human two-way MLR, $3 \times 10^{6}$ human PBMCs were incubated with $3 \times 10^{6}$ HLA-DR nonshared allogeneic PBMCs in the presence or absence of wogonin $(1 \mu \mathrm{M})$ in RPMI 1640 medium supplemented with $10 \%$ human serum in a flat-bottomed 12-well plate for seven days. The culture supernatants were assayed for IFN- $\gamma$, IL-4, IL-5, and IL-17 using ELISA kits (R\&D systems, U.S.A.).

\subsection{DC-Mediated T-Cell Differentiation Assay (One-Way} $M L R)$. For the human one-way MLR, $1 \times 10^{4}$ human immature Mo-DCs were seeded in a round-bottomed 96-well plate and then incubated in the presence or absence of wogonin $(1 \mu \mathrm{M})$ in $100 \mu \mathrm{L}$ of RPMI 1640 medium containing $10 \%$ human serum for two days. Following incubation, the MoDCs were washed once and then cocultured with $1 \times 10^{5}$ HLA-DR nonshared allogeneic $\mathrm{CD}^{+}$naive $\mathrm{T}$ cells in RPMI 1640 medium supplemented with $10 \%$ human serum in a round-bottomed 96-well plate for seven days. At seven days after incubation, the cells were washed twice and stimulated with anti-human CD3 and anti-human CD28 antibodies (BD Pharmingen, San Diego, U.S.A.) for 24 h. The supernatant was then collected for the IFN- $\gamma$, IL-4, IL-5, and IL-17 ELISA.

\subsection{Induction of Airway Inflammation and Administration} of Wogonin. Six- to ten-week-old female DO11.10 mice were challenged with an aerosolized solution of 3\% OVA (SigmaAldrich, St. Louis, U.S.A.) or PBS for $10 \mathrm{~min}$ from days -2 to 0 . From days -7 to 0 , some mice received wogonin $(2 \mathrm{mg} / \mathrm{kg} /$ day; Tsumura) in distilled water $(100 \mu \mathrm{L})$ or the vehicle orally every day. The mice were sacrificed, and the degree of neutrophilic airway inflammation was evaluated on day 1 .

2.7. Bronchoalveolar Lavage Fluid (BALF) Analyses. The BALF analyses were performed as previously reported [12]. The mice were anesthetized via intraperitoneal injection of sodium pentobarbital $(50 \mathrm{mg} / \mathrm{kg})$. Then, the lungs were lavaged four times with PBS $(0.5 \mathrm{~mL}$ each). Approximately $1.6 \mathrm{~mL}$ of the instilled PBS was consistently recovered with gentle handling. The cell suspension was centrifuged at $150 \times \mathrm{g}$ 

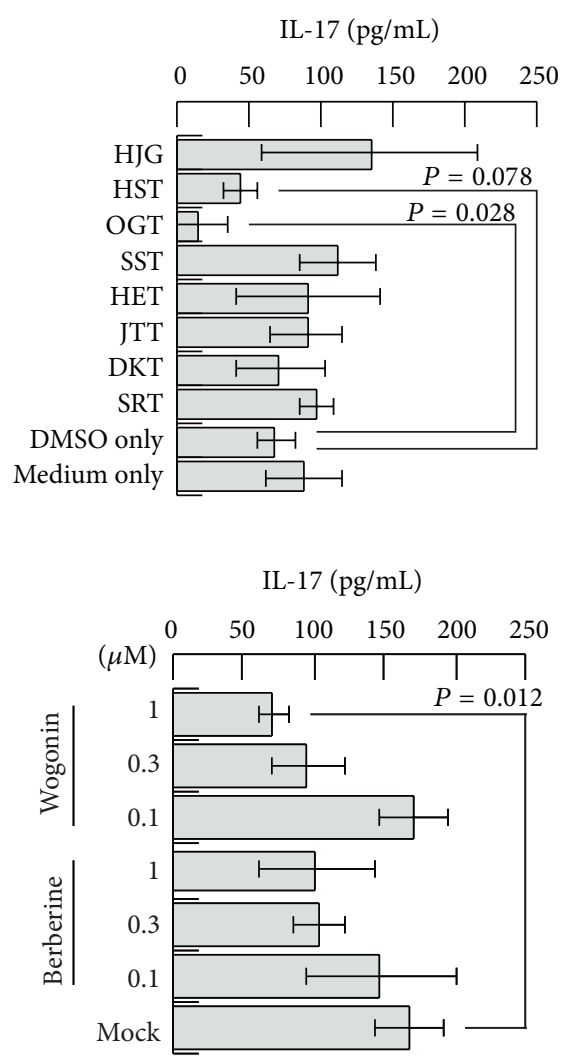

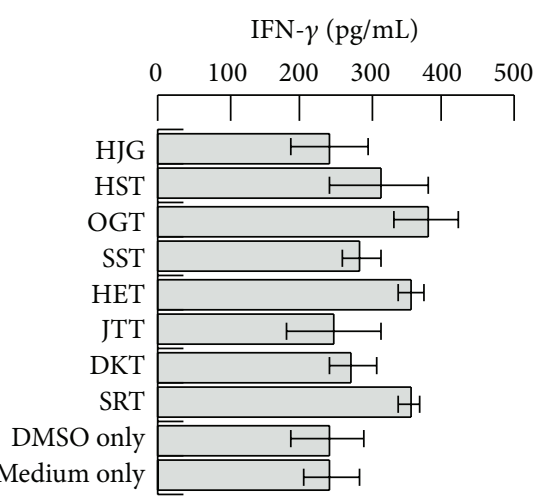

(a)

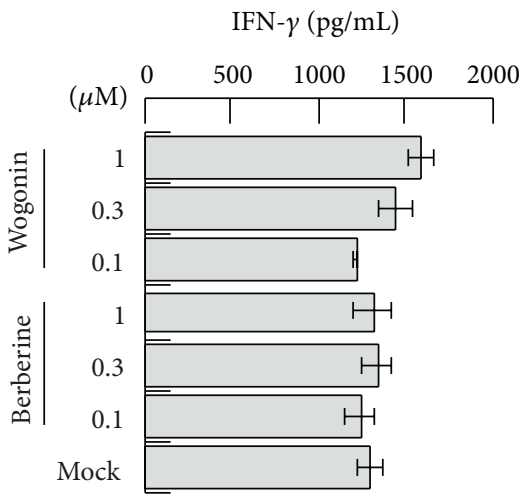

(b)
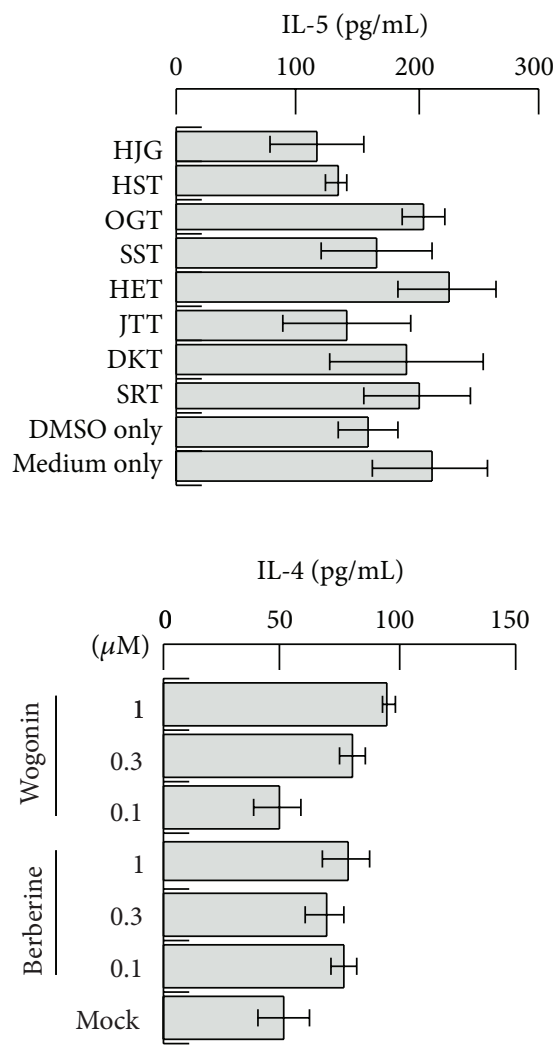

FIGURE 1: Screening of Kampo extracts and identification of major components that exhibit the inhibitory effects of IL-17 secretion. HJG, HST, OGT, SST, HET, JTT, DKT, or SRT were added at a concentration of $10 \mu \mathrm{g} / \mathrm{mL}$ to the two-way MLR of splenic lymphocytes obtained from BALB/c and SJL mice and then were incubated for seven days (a). The supernatants were analyzed using ELISA to detect the typical cytokine secretions of IFN- $\gamma$ (center), IL-5 (right), and IL-17 (left) for Th1, Th2, and Th17, respectively. Increasing concentrations of wogonin or berberine $(0.1,0.3$, and $1 \mu \mathrm{M})$ were mixed with a culture of lymphocytes obtained from BALB/c and SJL mice and then incubated for seven days (b). The supernatants were analyzed using ELISA to detect the typical cytokines of IFN- $\gamma$ (center), IL-4 (right), and IL-17 (left) for Th1, Th2, and Th17, respectively.

at $4^{\circ} \mathrm{C}$ for $10 \mathrm{~min}$. The cells were resuspended in $1 \mathrm{~mL}$ of PBS, and the total number of cells was counted with a hemocytometer. Cytospin samples were prepared by centrifuging the suspensions at $300 \mathrm{rpmfor} 10 \mathrm{~min}$. Based on the findings of May-Grünwald-Giemsa staining, the cell differentials were counted with at least 300 leukocytes in each sample. The cell types were judged according to standard hemocytologic procedures to be either neutrophils, eosinophils, lymphocytes, or macrophages.

2.8. Histological Examination. The histological examination was performed as previously reported [9]. Following perfusion with PBS, the right lungs were resected, fixed with $10 \%$ neutralized buffered formalin (Wako, Osaka, Japan), and embedded in paraffin. Three-micrometer-thick sections were stained with hematoxylin and eosin.

\subsection{Cytokine Production and Proliferation Assay of Human} Th17 Clones. Human alloreactive Th17 cell clones established in our previous study were used [17]. To analyze the cytokine production, Th17 cells were cocultured with irradiated allogeneic PBMCs in medium containing RPMI
1640 and $10 \%$ human serum and then were incubated for $24 \mathrm{~h}$ in the presence or absence of $1 \mu \mathrm{M}$ of wogonin. Following incubation, the supernatants were analyzed using an IL-17 ELISA. To assess the proliferation of the human Th17 clones, the cells were incubated with irradiated allogeneic PBMCs in the presence or absence of $1 \mu \mathrm{M}$ of wogonin for $72 \mathrm{~h}$ in the presence of $1 \mu \mathrm{Ci} /$ well of $\left[{ }^{3} \mathrm{H}\right] \mathrm{TdR}$ during the final $16 \mathrm{~h}$ period, and the incorporated radioactivity was measured using liquid scintillation counting after harvesting.

2.10. Statistics. The values are expressed as the mean \pm SEM. The statistical analyses were performed using a one-way ANOVA followed, when the differences were significant, by appropriate post hoc tests with the Tukey-Kramer test. To analyze differences between the two groups, we used Student's $t$-test. Values of $P<0.05$ were considered to be statistically significant.

\section{Results}

3.1. Screening of Kampo Extracts to Identify the Inhibitory Effects on Th17. In order to identify the compounds in 

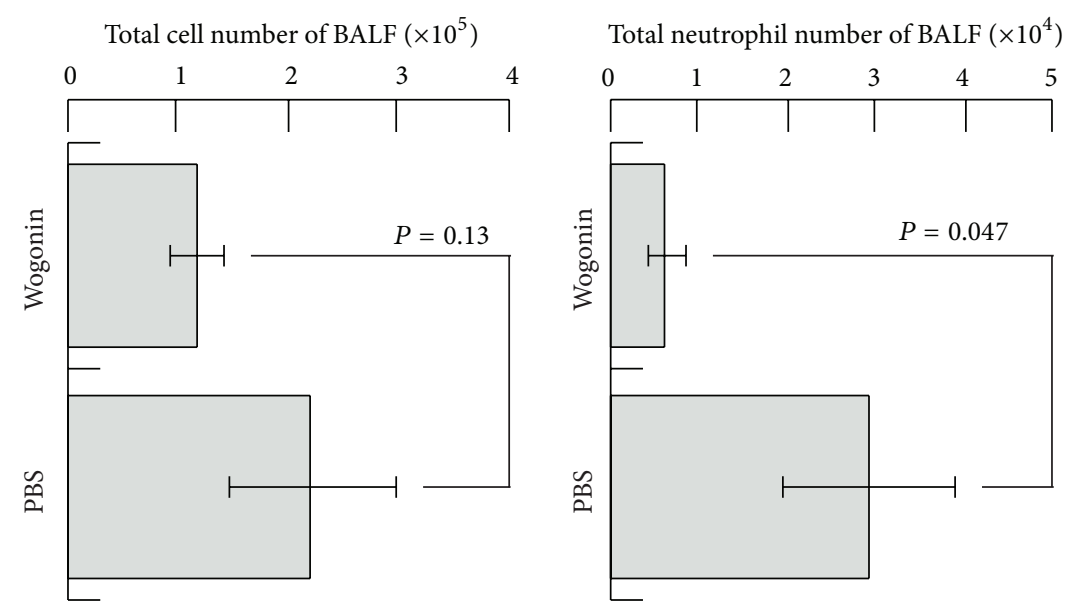

(a)
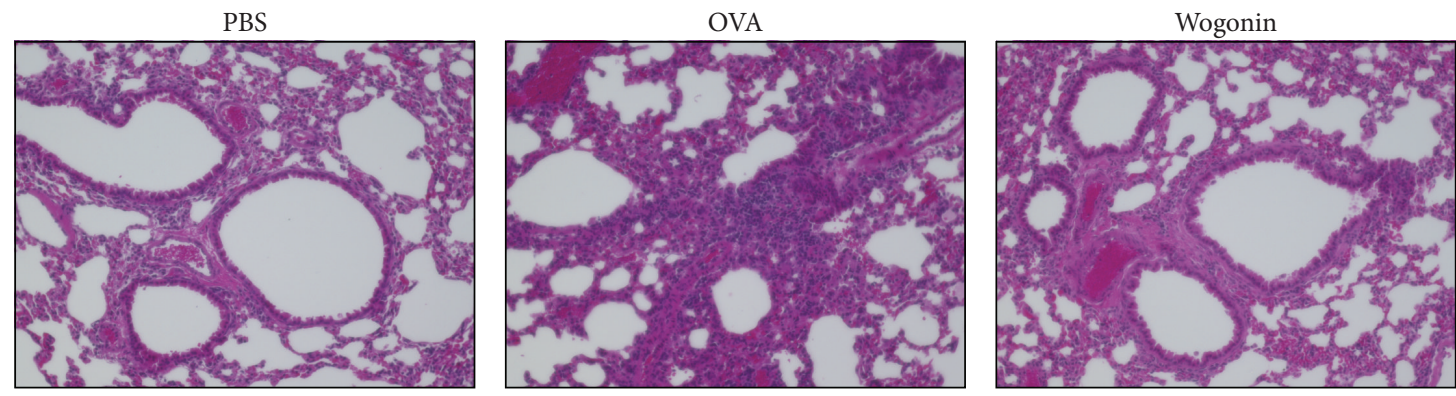

(b)

FIGURE 2: Decline in the number of neutrophils infiltrating the bronchi and lungs induced by wogonin treatment. DO11.10 mice were nebulized with either OVA or PBS from days -2 to 0 . Some mice received wogonin $(2 \mathrm{mg} / \mathrm{kg})$ or distilled water orally five times a week from days -7 to 0 . On day 1 , the mouse BALF was collected in order to count the number of total cells ((a), left) and neutrophils ((a), right). The mouse lungs were excised and subjected to hematoxylin and eosin staining (b). Histological staining was performed on the lungs removed from the mice that received water and were nebulized with PBS (left panel), the mice that received water and were nebulized with OVA (center panel), and the mice that received wogonin and were nebulized with OVA (right panel). Original magnification: $\times 200$.

the Kampo extracts that attenuate IL-17 production during the immune response, eight Kampo extracts were subjected to mouse two-way MLR, and the concentrations of the cytokines secreted during the immune reactions were evaluated. For this purpose, spleen cells obtained from BALB/c and SJL mice were mixed in the presence or absence of the Kampo extracts, and seven days after the initiation of the culture, the supernatants were collected and subjected to ELISA for cytokines. As shown in Figure 1(a), OGT significantly suppressed the IL-17 production. Although the $P$ value was more than 0.05 , HST also reproducibly suppressed the IL-17 production. The experiments were repeated three times, and the same results were obtained in each experiment. Wogonin and berberine are the major components common to the two Kampo extracts and were therefore evaluated in the further assays.

3.2. Activity of Wogonin in the Mouse Two-Way MLR. We therefore evaluated the activity of wogonin and berberine during the immune reactions in the mouse two-way MLR (Figure 1(b)). As expected, wogonin significantly suppressed the IL-17 production at a concentration of $1 \mu \mathrm{M}$. Although the effects were lower in magnitude, berberine also suppressed the IL-17 production at a concentration of $1 \mu \mathrm{M}$. The experiments were repeated twice, and the same results were obtained each time.

3.3. Activity of Wogonin in the Neutrophilic Airway Inflammation Model. To elucidate the effects of wogonin in vivo, an OVA-induced neutrophilic airway inflammation model using DO11.10 mice was employed [9]. The administration of wogonin was performed starting six days before OVA nebulization. OVA nebulization markedly increased the number of neutrophils in the BALF of the DO11.10 mice, while the increase in the level of neutrophils was significantly suppressed by wogonin treatment (Figure 2(a)). The histology of the OVAchallenged DO11.10 mice demonstrated marked congestion and prominent neutrophil infiltration in the peribronchial areas (Figure 2(b)). In contrast, the degree of infiltration was markedly lower in the mice that received wogonin. The experiments were repeated twice, and the same results were obtained each time. These results suggest that wogonin has an effect on neutrophil-mediated airway inflammation via Th17 suppression. 

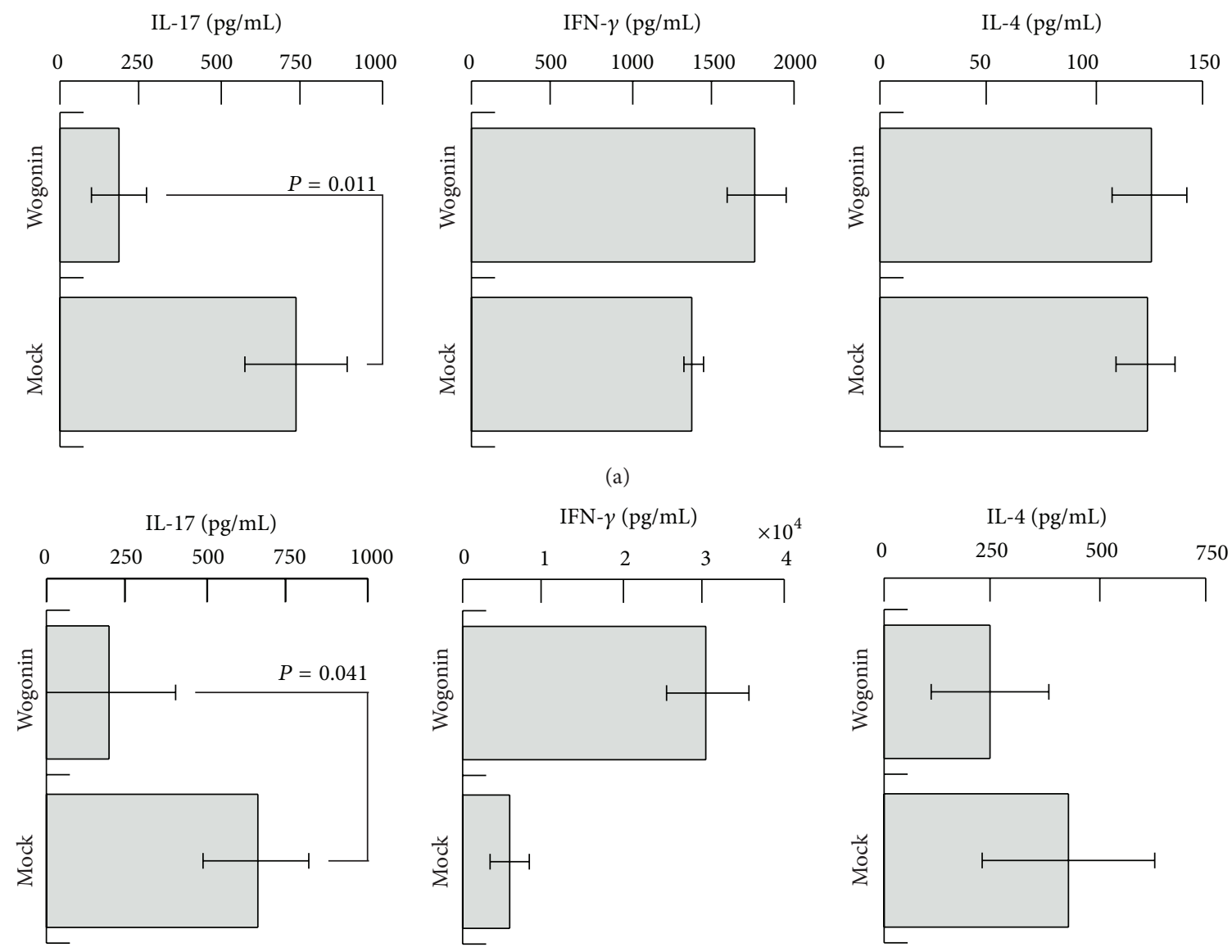

(b)

Figure 3: Suppression of IL-17 by wogonin treatment in the human MLR. For the human two-way MLR (a), wogonin ( $1 \mu \mathrm{M})$ was exposed to a mixture of human allogeneic lymphocytes and then incubated for seven days. Seven days after incubation, the supernatants were analyzed using ELISA to detect the typical cytokines of IFN- $\gamma$ (center), IL-4 (right), and IL-17 (left) for Th1, Th2, and Th17, respectively. For the human one-way MLR (b), wogonin $(1 \mu \mathrm{M})$ was incubated with human Mo-DCs for two days. Then, the wogonin-treated human Mo-DCs were incubated with human allogeneic naïve $\mathrm{CD}_{4}^{+} \mathrm{T}$ cells in the absence of wogonin for seven days. The cells were restimulated with anti-human CD3 and CD28 antibodies for $24 \mathrm{~h}$, and the supernatants were collected for ELISA to detect the typical cytokines of IFN- $\gamma$ (center), IL- 4 (right), and IL-17 (left) for Th1, Th2, and Th17, respectively.

3.4. Effects of Wogonin in the Human MLR. To evaluate the effects of wogonin on the human immune system, a human two-way MLR was performed (Figure 3(a)). Again, wogonin exhibited suppressive activity against IL-17 production. The production of IL- 4 was not significantly affected. These observations collectively indicate that wogonin suppresses the IL-17 production during immune responses in both mouse and human systems, raising the possibility that wogonin inhibits DC-mediated Th17 differentiation. To address this issue, wogonin was subjected to a human one-way MLR (Figure 3(b)). Human monocyte-derived immature DCs were incubated with wogonin for two days and then were incubated with human allogeneic naïve $\mathrm{CD} 4^{+} \mathrm{T}$ cells in the absence of wogonin. Seven days after incubation, the cells were stimulated with anti-human CD3 and anti-human CD28 antibodies for $24 \mathrm{~h}$. The supernatant was then collected and analyzed using ELISA to assess the production of IL17 , IFN- $\gamma$, and IL- 4 . As expected, the production of IL-17 was suppressed by the treatment of immature DCs with wogonin, suggesting that wogonin inhibits DC-mediated Th17 differentiation.

3.5. Wogonin Does Not Inhibit the Secretion of IL-17 or the Proliferation of Th17 Cells. In order to exclude the possibility that wogonin directly inhibits the production of IL17 and proliferation of Th17 cells, cloned human Th17 cells established in our previous study [17] were cocultured with wogonin (Figure 4(a)). Consequently, neither IL-17 production nor proliferative responses were affected. The experiment was repeated once, and the same results were obtained. All of these results collectively indicate that wogonin does not affect activated Th17 cells, but rather abrogates the DC-mediated differentiation of naïve $\mathrm{T}$ cells to Th17 cells.

\section{Discussion}

Recently, accumulated data on immunological disorders have indicated that the generation of Th17 is closely associated with 


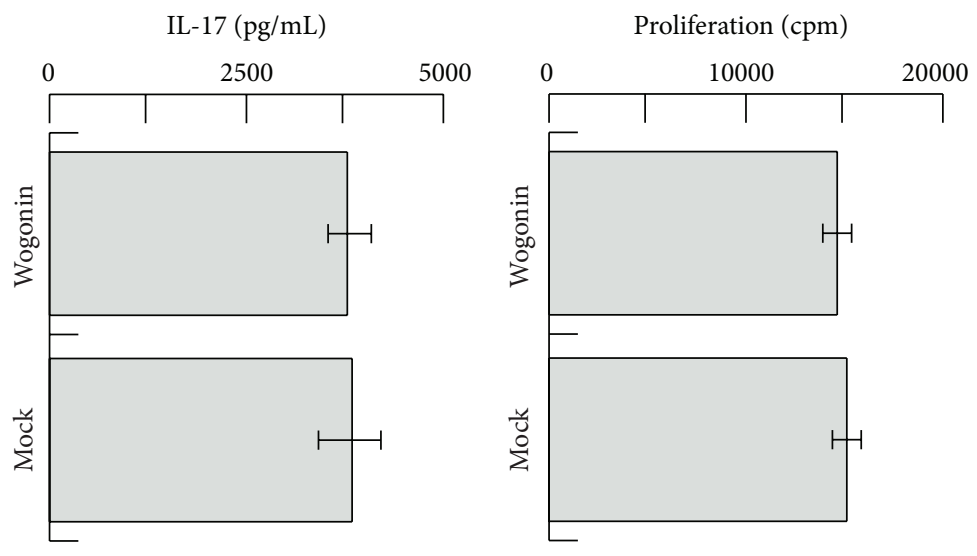

(a)

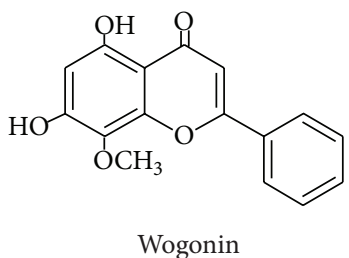

(b)

FIGURE 4: Effects of wogonin on a human Th17 clone. The human alloreactive Th17 clone was established earlier [17]. Following incubation of Th17 with irradiated allogeneic PBMCs in the presence or absence of wogonin $(1 \mu \mathrm{M})$ for $24 \mathrm{~h}$, the supernatants were analyzed using ELISA to detect the secretion of IL-17 ((a), left). To assess the proliferation of Th17, the cells were incubated with irradiated allogeneic PBMCs in the presence or absence of wogonin $(1 \mu \mathrm{M})$ for $72 \mathrm{~h}$ and analyzed using $\left[{ }^{3} \mathrm{H}\right] \mathrm{TdR}$ incorporation during the final $16 \mathrm{~h}$ period. The incorporated radioactivity was analyzed using liquid scintillation counting after harvesting ((a), right). Wogonin belongs to flavonoid species (b).

the development of many autoimmune conditions [18]. Since IL-17 induces chemoattraction of neutrophils, the activation of Th17 for IL-17 secretion is speculated to exacerbate the neutrophilic inflammation observed in many autoimmune diseases. It is well known that several Kampo medicines exhibit efficacy in suppressing inflammation, including that observed in gastritis, colitis, arthritis, hepatitis, pneumonia, and dermatitis. However, the detailed mechanisms underlying the anti-inflammatory effects of these Kampo medicines remain largely unknown. We presumed that the compounds contained in Kampo extracts would inhibit Th17 generation. In order to identify such molecules, we decided to use our established systems to analyze the Th1-Th2-Th17 balance and adjuvant activities.

Eight kinds of Kampo extracts were initially examined in our screening assay to identify which Kampo extracts are capable of inhibiting the IL-17 production. OGT significantly reduced the allogeneic antigen-induced production of IL-17, but not that of IL-4 or IFN- $\gamma$. Moreover, HST also showed a tendency to reduce IL-17, although the inhibitory effect of HST was less than that of OGT. OGT is an extract of a mixture of the following four medicinal herbs in the ratios provided in the parentheses: Scutellaria root (3.0), Coptis rhizome (2.0), Gardenia fruit (2.0), and Phellodendron bark (1.5). HST is an extract of a mixture of the following seven medicinal herbs in the ratios provided in the parentheses: Scutellaria root (2.5), Coptis rhizome (1.0), Pinellia tuber (5.0), Processed ginger (2.5), Glycyrrhiza (2.5), Jujube (2.5), and Ginseng (2.5). Therefore, both OGT and HST have common components originating from Scutellaria root and Coptis rhizome. In addition, wogonin and berberine are more abundant in OGT than in HST because the constitutive ratios of the two common medicinal herbs in OGT are higher than those observed in HST. The other six kinds of Kampo extracts that demonstrated no effects on the IL-17 production do not contain Coptis rhizome. The SST and SRT extracts contain
Scutellaria root, although at an extremely lower amount than OGT or HST, considering the constitutive ratios of the two medicinal herbs. The present study indicates that wogonin and berberine exhibit strong and selective activities to inhibit the allogeneic antigen-induced IL-17 production. Furthermore, we revealed that wogonin suppressed DCmediated Th17 differentiation in a human one-way MLR. On the other hand, in the human Th17 clone, wogonin had no effect on the secretion of IL-17 or proliferation. Overall, the results suggest that wogonin affects DCs rather than alreadydifferentiated Th17 cells in the suppression of IL-17 secretion.

Previously, a dopamine D1-like-R antagonist, SCH23390, was found to significantly improve neutrophilic airway inflammation induced by OVA administration [9], suggesting that the inhibitory activity of wogonin against D1-Rmediated signaling may also improve neutrophilic airway inflammation. We therefore attempted to show the activity of wogonin by using D1R antagonism assay, D1R binding assay, and tyrosine hydroxylase inhibition assay. However, all these experiments gave negative results (not shown).

Wogoin belongs to flavonoids (Figure 4(b)). In this relation, Morita, et al. demonstrated that other flavonoids inhibited the release of catecholamines [19], which should also be tested with wogonin. It is also possible to speculate that wogonin may interact directly with naive $\mathrm{T}$ cells, because both DCs and T cells are exposed to wogonin, in two-way MLR assay shown in our current study. In this case, molecules such as Akt, mTOR, HIF-1 $\alpha$, and STAT3 expressed in naive T cells need to be considered as targets for wogonin [20]. The study is currently underway.

HST and OGT have classically been shown to harbor anxiolytic effects, including neurosis and insomnia. From this point of view, wogonin also has been shown to exert anxiolytic effects in the screening of components of HST and OGT [21]. In parallel, Kampo extracts have an effect on cancer prevention $[22,23]$, and wogonin has been clarified 
to have an antitumor effect in bladder cancer cell lines [24]. Additionally, wogonin inhibits the glioma-mediated induction of the Treg phenotype via inhibition of the TGF- $\beta 1$ activity [25]. Immunomodulatory effect of wogonin on virus infection [26] and LPS stimulation [27] has been observed; however, the effects of wogonin on Th1-Th2-Th17 balance have not been considered to date. This is the first report, to our knowledge, to show that wogonin has the potency to reduce the Th17 population, which should greatly contribute to the effective treatment of autoimmune disorders. Although increasing evidence has shown the cytotoxicity of wogonin in multiple cancer cell lines $[28,29]$, cell viability was not affected by coculture with wogonin in MLR experiments (data not shown).

\section{Conclusions}

Taken together, the findings of the current study demonstrated that wogonin has the potency to selectively suppress DC-mediated Th17 differentiation. Our study suggests that wogonin may, therefore, be an effective therapeutic agent for Th17-driven diseases and its use may thus be useful as a new strategy to treat neutrophilic inflammatory disorders.

\section{Conflict of Interests}

The authors declare that there is no conflict of interests regarding the publication of this paper. Sho Matsushita is an employee of iMmno, Inc.

\section{Acknowledgments}

This work was partially funded by Tsumura and Co. and was also supported by MEXT KAKENHI, Grant nos. 23591466 and 22790940.

\section{References}

[1] T. Korn, E. Bettelli, M. Oukka, and V. K. Kuchroo, "IL-17 and Th17 cells," Annual Review of Immunology, vol. 27, pp. 485-517, 2009.

[2] R. S. Liblau, S. M. Singer, and H. O. McDevitt, "Th1 and Th2 CD4+ T cells in the pathogenesis of organ-specific autoimmune diseases," Immunology Today, vol. 16, no. 1, pp. 34-38, 1995.

[3] A. Sher and R. L. Coffman, "Regulation of immunity to parasites by $\mathrm{T}$ cells and T cell-derived cytokines," Annual Review of Immunology, vol. 10, pp. 385-409, 1992.

[4] P. Kaliński, C. M. U. Hilkens, E. A. Wierenga, and M. L. Kapsenberg, "T-cell priming by type- 1 and type- 2 polarized dendritic cells: the concept of a third signal," Immunology Today, vol. 20, no. 12, pp. 561-567, 1999.

[5] S. Matsushita, R. Takagi, K. Hashimoto, and T. Higashi, "Qualitative evaluation of adjuvant activities and its application to Th2/17 diseases," International Archives of Allergy and Immunology, vol. 155, no. 1, pp. 2-5, 2011.

[6] T. Higashi, M. Wakui, K. Nakano et al., "Evaluation of adjuvant activities using human antigen presenting cells in vitro," Allergology International, vol. 57, no. 3, pp. 219-222, 2008.
[7] T. Higashi, K. Hashimoto, R. Takagi et al., "Curdlan induces DC-mediated Th17 polarization via Jaggedl activation in human dendritic cells," Allergology International, vol. 59, no. 2, pp. 161166, 2010.

[8] K. Nakano, K. Yamaoka, K. Hanami et al., "Dopamine induces IL-6-dependent IL-17 production via D1-like receptor on CD4 naive $\mathrm{T}$ cells and D1-like receptor antagonist SCH-23390 inhibits cartilage destruction in a human rheumatoid arthritis/SCID mouse chimera model," Journal of Immunology, vol. 186, no. 6, pp. 3745-3752, 2011.

[9] K. Nakagome, M. Imamura, H. Okada et al., "Dopamine D1-like receptor antagonist attenuates Th17-mediated immune response and ovalbumin antigen-induced neutrophilic airway inflammation," Journal of Immunology, vol. 186, no. 10, pp. 59755982, 2011.

[10] A. T. Borchers, S. Sakai, G. L. Henderson et al., "Shosaiko-to and other Kampo (Japanese herbal) medicines: a review of their immunomodulatory activities," Journal of Ethnopharmacology, vol. 73, no. 1-2, pp. 1-13, 2000.

[11] J. J. Gao, P. P. Song, F. H. Qi, N. Kokudo, X. J. Qu, and W. Tang, "Evidence-based research on traditional Japanese medicine, Kampo, in treatment of gastrointestinal cancer in Japan," Drug Discoveries \& Therapeutics, vol. 6, no. 1, pp. 1-8, 2012.

[12] H. Suzuki, J. M. Inadomi, and T. Hibi, "Japanese herbal medicine in functional gastrointestinal disorders," Neurogastroenterology and Motility, vol. 21, no. 7, pp. 688-696, 2009.

[13] Y. Kase, M. Yuzurihara, S. Iizuka, A. Ishige, and Y. Komatsu, "The effects of Hange-shashin-to on gastric function in comparison with Sho-saiko-to," Biological and Pharmaceutical Bulletin, vol. 20, no. 11, pp. 1155-1159, 1997.

[14] K. Kawashima, A. Nomura, T. Makino, K.-I. Saito, and Y. Kano, "Pharmacological properties of traditional medicine (XXIX): effect of hange-shashin-to and the combinations of its herbal constituents on rat experimental colitis," Biological and Pharmaceutical Bulletin, vol. 27, no. 10, pp. 1599-1603, 2004.

[15] Y. Ohta, T. Kobayashi, K. Nishida, E. Sasaki, and I. Ishiguro, "Preventive effect of Oren-gedoku-to (Huanglian-Jie-Du-Tang) extract on the development of stress-induced acute gastric mucosal lesions in rats," Journal of Ethnopharmacology, vol. 67, no. 3, pp. 377-384, 1999.

[16] H. Zhou and S. Mineshita, "The effect of Oren-gedoku-to on experimental colitis in rats," Journal of Pharmacy and Pharmacology, vol. 51, no. 9, pp. 1065-1074, 1999.

[17] R. Takagi, T. Higashi, K. Hashimoto et al., "B Cell chemoattractant CXCL13 is preferentially expressed by human Th17 cell clones," Journal of Immunology, vol. 181, no. 1, pp. 186-189, 2008.

[18] J. C. Waite and D. Skokos, “Th17 response and inflammatory autoimmune diseases," International Journal of Inflammation, vol. 2012, Article ID 819467, 2012.

[19] K. Morita, S. Hamano, K. Teraoka, M. Oka, and M. Yoshizumi, "Effects of bioflavonoids on exocytotic releasee of catecholamines from digitonin-permeabilized chromaffin cells: a comparison with the effects of other protein kinase C inhibitors," Neurochemistry International, vol. 16, no. 3, pp. 313318, 1990.

[20] Y. Kurebayashi, S. Nagai, A. Ikejiri, and S. Koyasu, "Recent advances in understanding the molecular mechanisms of the development and function of Th17 cells," Genes to Cells, vol. 18, no. 4, pp. 247-265, 2013.

[21] K. M. Hui, M. S. Y. Huen, H. Y. Wang et al., "Anxiolytic effect of wogonin, a benzodiazepine receptor ligand isolated from 
Scutellaria baicalensis Georgi," Biochemical Pharmacology, vol. 64, no. 9, pp. 1415-1424, 2002.

[22] M. Tatsuta, H. Iishi, M. Baba, A. Nakaizumi, and H. Uehara, "Inhibition of Xiao-chai-hu-tang (TJ-9) of development of hepatic foci induced by $\mathrm{N}$-nitrosomorpholine in Sprague-Dawley rats," Japanese Journal of Cancer Research, vol. 82, no. 9, pp. 987992, 1991.

[23] H. Yano, A. Mizoguchi, K. Fukuda et al., "The herbal medicine sho-saiko-to inhibits proliferation of cancer cell lines by inducing apoptosis and arrest at the G0/G1 phase," Cancer Research, vol. 54, no. 2, pp. 448-454, 1994.

[24] S. Ikemoto, K. Sugimura, N. Yoshida et al., "Antitumor effects of Scutellariae radix and its components baicalein, baicalin, and wogonin on bladder cancer cell lines," Urology, vol. 55, no. 6, pp. 951-955, 2000.

[25] S. Dandawate, L. Williams, N. Joshee et al., "Scutellaria extract and wogonin inhibit tumor-mediated induction of $\mathrm{T}$ reg cells via inhibition of TGF- $\beta 1$ activity," Cancer Immunology, Immunotherapy, vol. 61, no. 5, pp. 701-711, 2012.

[26] Z. Błach-Olszewska, B. Jatczak, A. Rak et al., "Production of cytokines and stimulation of resistance to viral infection in human leukocytes by Scutellaria baicalensis flavones," Journal of Interferon and Cytokine Research, vol. 28, no. 9, pp. 571-581, 2008.

[27] I. Wakabayashi and K. Yasui, "Wogonin inhibits inducible prostaglandin E2 production in macrophages," European Journal of Pharmacology, vol. 406, no. 3, pp. 477-481, 2000.

[28] S. Baumann, S. C. Fas, M. Giaisi et al., "Wogonin preferentially kills malignant lymphocytes and suppresses T-cell tumor growth by inducing PLC $\gamma 1-$ and $\mathrm{Ca}^{2+}$-dependent apoptosis," Blood, vol. 111, no. 4, pp. 2354-2363, 2008.

[29] G. Polier, J. Ding, B. V. Konkimalla et al., "Wogonin and related natural flavones are inhibitors of CDK9 that induce apoptosis in cancer cells by transcriptional suppression of Mcl-1," Cell Death \& Disease, vol. 2, no. 7, article e182, 2011. 


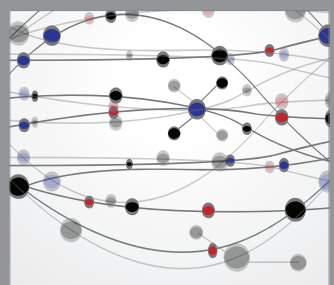

The Scientific World Journal
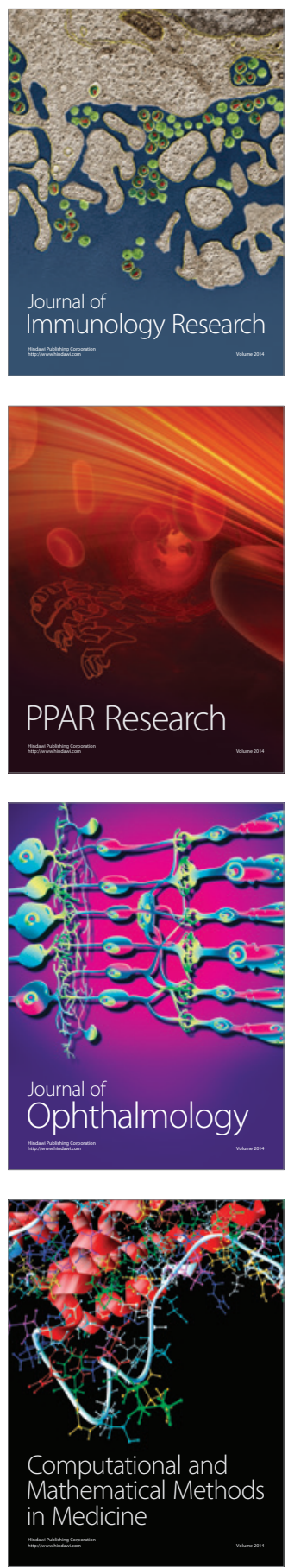

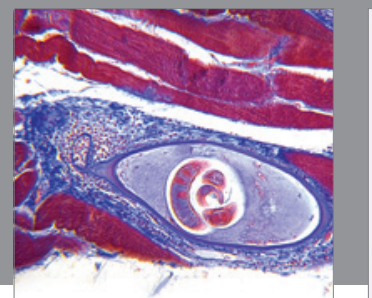

Gastroenterology

Research and Practice
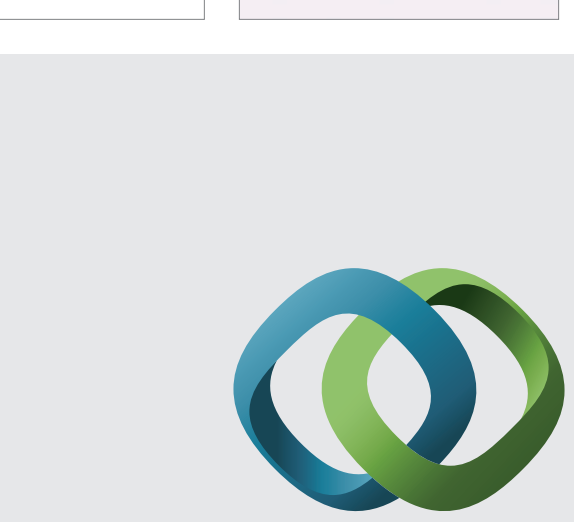

\section{Hindawi}

Submit your manuscripts at

http://www.hindawi.com
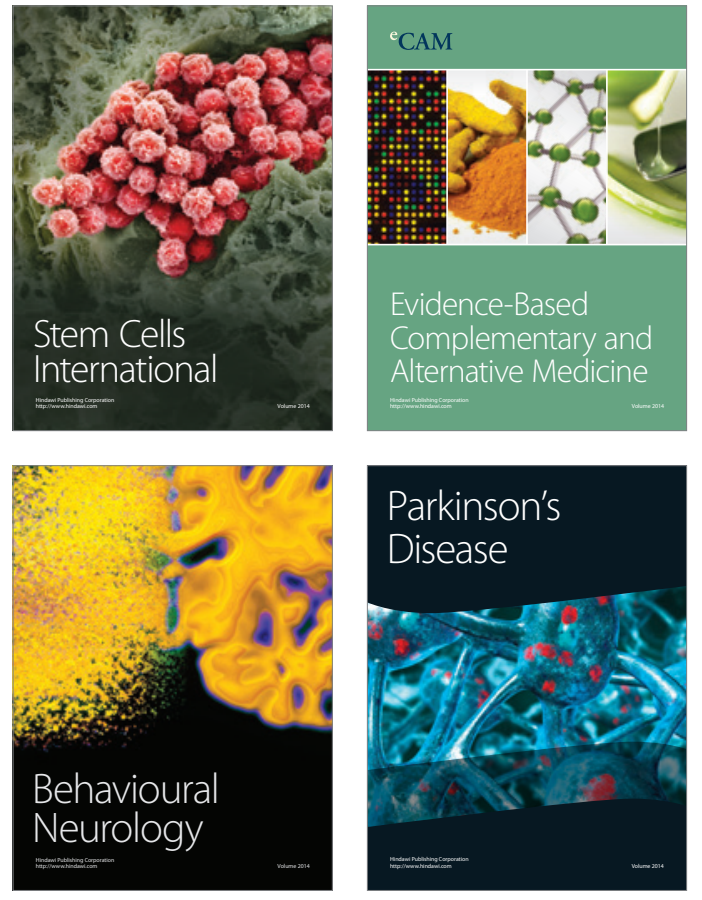
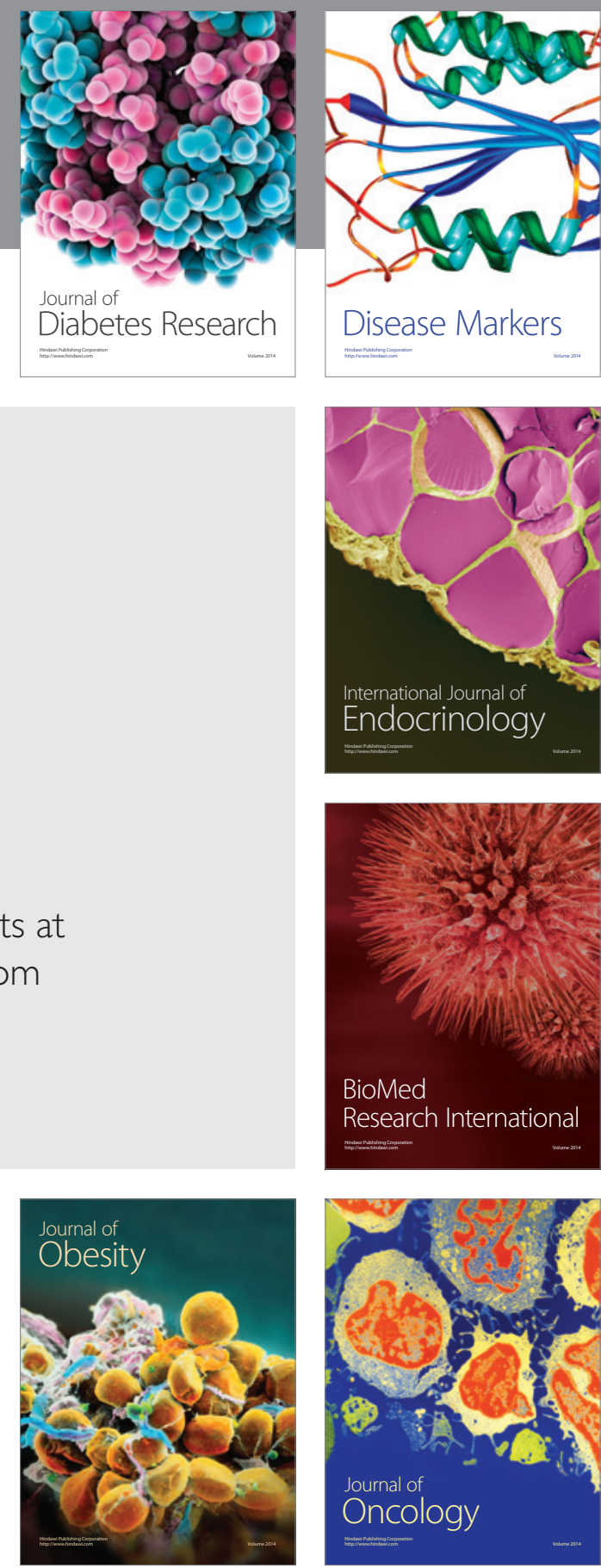

Disease Markers
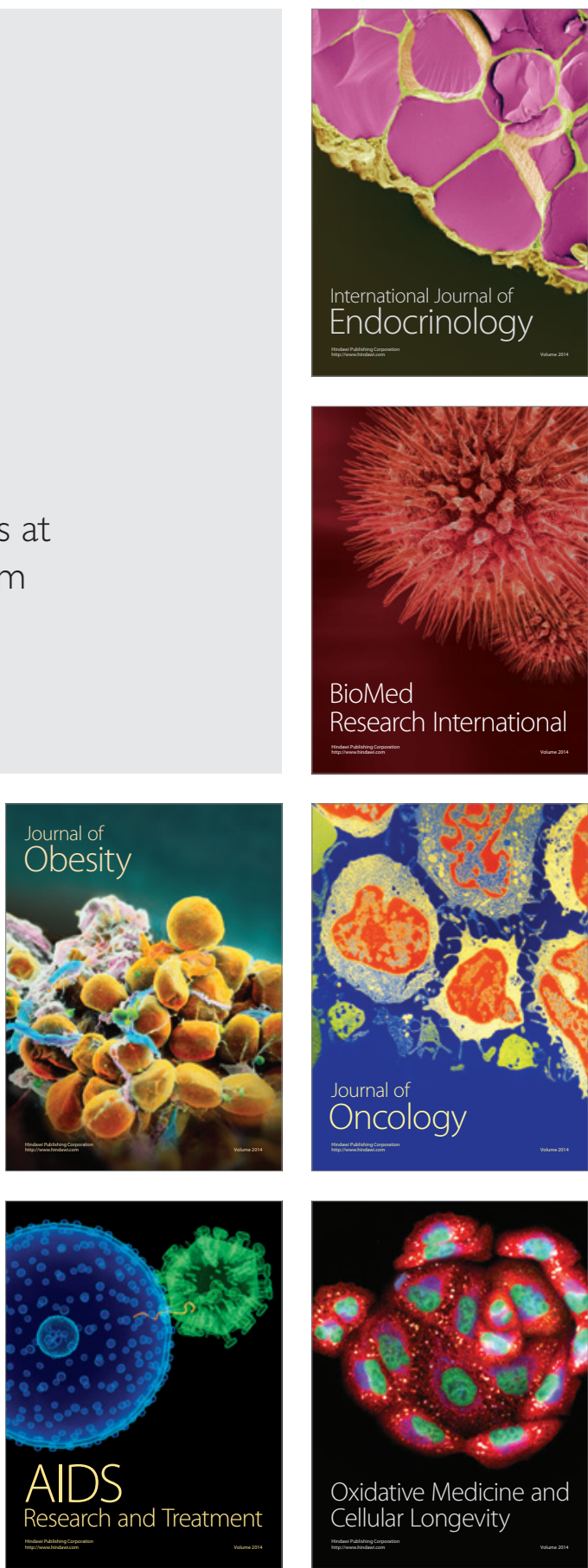